\title{
Light propagation in optical waveguides: a dynamic programming approach
}

\author{
Maria Luisa Calvo \\ Departamento de Optica, Facultad de Ciencias Fisicas, Universidad Complutense, 28040 Madrid, Spain
}

Vasudevan Lakshminarayanan

School of Optometry and Department of Physics and Astronomy, University of Missouri-St. Louis, 8001 Natural Bridge Road, St. Louis, Missouri 63121-4499

\begin{abstract}
Received February 22, 1996; revised manuscript received October 23, 1996; accepted November 19, 1996
We apply techniques of optimal-control theory, namely, the methods of dynamic programming, to the problem of light propagation in optical waveguides. This formulation is equivalent to the resolution of an eikonal equation. We illustrate this optimization technique for the case of an ideal parabolic refractive-index-profile distribution. We discuss the possibility of extending this procedure to other types of optical waveguides and optical media. @ 1997 Optical Society of America [S0740-3232(97)00105-1]
\end{abstract}

\section{INTRODUCTION}

The use of optimization techniques to describe light propagation phenomena through optical media with different optical properties and geometry is at present a relatively new area of investigation. This methodology concerns optimized design of arbitrary optical media for which an a priori characterization of significant parameters would be required. The problem of characterizing the output of a system from partial input data is defined as a control theoretical problem. To the best of our knowledge, this approach has not been used to study light propagation. (Other relevant applications include optical system design, laser cavities, laser beam modification, minimizing of geometrical aberrations, etc.)

Currently, optimization techniques applied to waveguide design are based on variational analysis related to the Sturm-Liouville theory and the Rayleigh-Ritz method. ${ }^{1}$ Recently, for example, Sharma and Bindal applied such a technique to optimize diffuse planar and channel waveguides. $^{2}$ Another approach that considerably simplifies this analysis is the well-known ray-tracing technique. $^{3}$ Ray trajectories are solutions from the eikonal approach to the partial differential equation for the electromagnetic field propagating inside an optical medium. This technique has the restrictions inherent in the integration conditions of the ray-path integral and in the required initial ray conditions. Also, an inverse procedure is not always applicable, for obvious reasons. One of the main problems in optimizing the ray-trajectory solution is the application of optimal interpolant procedures. This usually requires the implementation of computational methods, such as the Runge-Kutta, with the cost of the time and design of complex computational techniques. $^{4,5}$ The search for new simplified techniques requires a comparison between light propagation phenomena in inhomogeneous media and mechanical models. In this type of treatment the dynamic properties of refrac- tive phenomena are given in terms of two Hamiltonian equations. The solutions give both the vector position and the tangent to the trajectory. ${ }^{6}$ It should be noted that equivalent Lagrangian formulations for studying ray paths in cylindrically symmetric media have been proposed and have been applied to obtain exact ray paths in bent slabs as well as in bent fibers with separable profiles. $^{7}$

Ghatak and Sauter ${ }^{8,9}$ have discussed the harmonicoscillator problem in the domains of classical and quantum mechanics and the parabolic-index optical-waveguide problem in the domains of ray and wave optics, using a Lagrangian formulation. They showed that the time evolution of a coherent state has a close relationship to the propagation of a Gaussian beam in a parabolic-index waveguide. With the aim of finding simple alternative models, we have applied the method of dynamic programming to light propagation in inhomogeneous media. There is a former contribution to this idea in the earlier paper of Kalaba, ${ }^{10}$ where it was demonstrated that the eikonal equation may be derived directly from Fermat's principle of least time by using Bellman's principle of optimality. ${ }^{11,12}$ In the present paper we analyze the procedure and extend it to inhomogeneous media with arbitrary refractive-index distribution.

The paper is organized as follows: Section 2 presents background with the fundamentals of the adaptivecontrol process. In Section 3 we revisit the dynamicprogramming approach to optical systems and demonstrate that the eikonal solution is a Hamilton-Jacobi-type equation. Specific solutions derive from the EulerLagrange formulation, which is obtained from initial conditions imposed on the system. Section 4 is dedicated to application of these results to homogeneous optical media and Section 5 to general cases of gradient-index optical waveguides. In Section 6 we analyze the case of a parabolic profile and present some numerical results. We 
compare the results for the ray transit time with those predicted by the geometrical optics approximation. Finally, Section 7 consists of discussion and conclusions resulting from the various methods.

\section{HISTORICAL BACKGROUND: FUNDAMENTALS OF THE MATHEMATICAL THEORY OF THE ADAPTIVE-CONTROL PROCESS}

The mathematical theory of the adaptive-control process was first introduced by Bellman in 1957. ${ }^{11}$ Later, Bellman and Kalaba ${ }^{13}$ and Dreyfus, ${ }^{14}$ as well as Bellman and Vasudevan $^{15}$ and others, ${ }^{16}$ extended the applications to a wide variety of problems in physics, chemistry, and biology as well as in engineering, economics, and management science. In brief, control theory determines the behavior of arbitrary systems through optimized output responses, $F_{\text {out }}$. Consider an arbitrary system represented by the action of an operator $S$ (linear or nonlinear); then $F_{\text {out }}=S\left[F_{\text {in }}\right]$, where $F_{\text {in }}$ is any arbitrary input. More-sophisticated representations are also feasible by introduction of a higher number of inputs interacting with diverse categories of systems and networks (a multistage control process).

In general, the mathematical framework used to solve a control process deals with the minimization of a quadratic functional ${ }^{17}$ of the form

$$
J(u, v)=\int^{T} \mathrm{~d} t\left[u^{2}(t)+v^{2}(t)\right],
$$

where $u(t)$ and $v(t)$ have a ligature and $T$ is an arbitrary time interval. In this case $u(t)$ and $v(t)$ might, for example, be related by a linear differential equation of the form

$$
\frac{\mathrm{d} u}{\mathrm{~d} t}=a u+v
$$

with the initial condition $u(0)=C \quad(C$ arbitrary constant).

This class of quadratic nonlinear functional is characteristic of equations derived by invariant imbedding techniques. ${ }^{17}$ Let us assume a system $S$ having a certain state at any time $t$. The description of this state is a first global constraint, $u=u(t)$, whose solution is determined by an initial condition. $u(t)$ is a scalar function whose physical meaning is a displacement. Its first derivative, $u^{\prime}=\mathrm{d} u / \mathrm{d} t$, has dimensions of velocity. In this way, one can determine other global constraints such as the acceleration or maybe other higher-order derivatives, by formulating a nonlinear differential equation (at least of the second degree):

$$
u^{\prime \prime}+u^{\prime}+u=g\left(u, u^{\prime}\right)
$$

In Eq. (3) the initial conditions are $u(0)=c_{1}$ and $u^{\prime}(0)$ $=c_{2} ; c_{1}, c_{2}$ constants.

One may search for an explicit solution for $g\left(u, u^{\prime}\right)$ by minimizing the quadratic functional:

$$
J\left(u, u^{\prime}\right)=\int^{T}\left(u^{2}+u^{\prime 2}\right) \mathrm{d} t .
$$

In the simplest case, the description of the system is with one single-state variable, the displacement $u(t)$, and the specific problem will be the minimization of the functional together with the initial condition $u(0)=c_{1}$. The solution is not always a trivial one. The most common and well-known methods are those based on linear partial differential equations and the calculus of variations. ${ }^{18,19}$ For example, a formal method would be through the Euler equation, and the uniqueness of the solution as a desired minimum has to be proven. A different approach to solving the functional is based on the theory of dynamic programming, defined as an extension of the feedbackcontrol process. ${ }^{10}$ The formalism of dynamic programming requires the definition of a minimum pathway. Direct application of Fermat's principle leads to the classical problem of solving an eikonal equation. Figure 1 displays the interrelationships schematically.

We now introduce the idea of "bang-bang" control. In bang-bang control theory the initial condition assigns bounded values to the origin of the trajectory (they are discrete and can be either positive or negative). Minimization of time is ensured by performing first an acceleration from the initial point up to an intermediate one and then a deceleration from this intermediate point up to the last position. For light propagation phenomena this procedure has no specific physical meaning, and one assumes only that it ensures a defined behavior for the slope, e.g., linear concerning the trajectory. We refer the readers to the many excellent texts on dynamic programming and adaptive-control process listed in the references for further details.

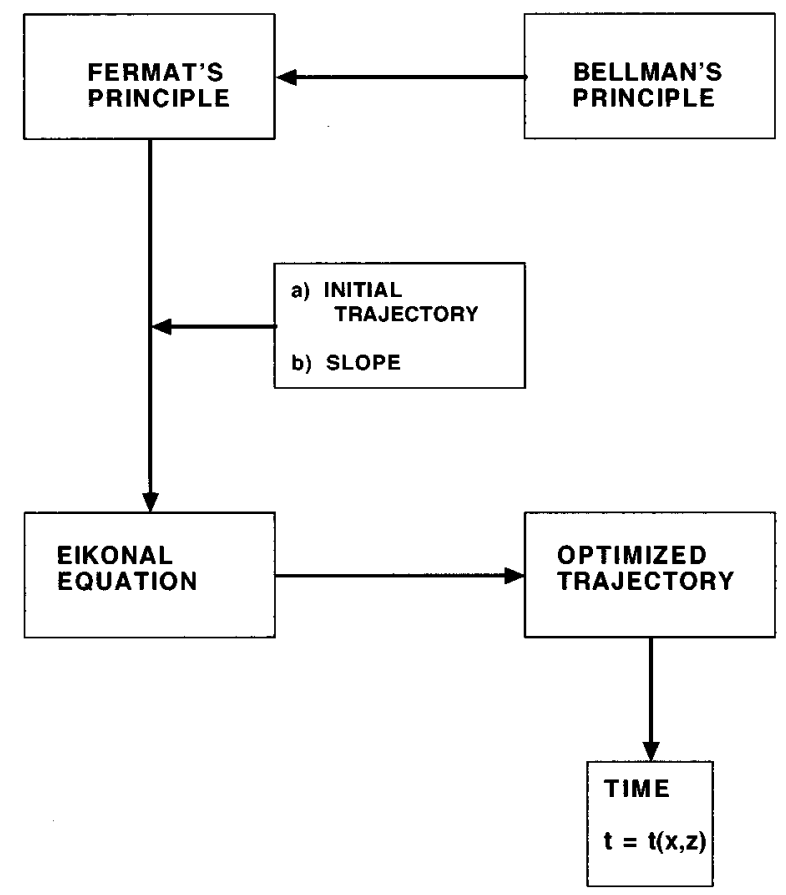

Fig. 1. Schematic diagram showing the connection between dynamic programming and optimized trajectory (time minimization) for light propagating in an arbitrary optical medium. 


\section{DYNAMIC PROGRAMMING REVISITED: GENERAL DESCRIPTION AND FORMULATION OF THE PROBLEM IN OPTICAL SYSTEMS}

Let us consider Fig. 2(a). The graph $z=z(x)$ represents an arbitrary path for light propagating from point $P(x, y)$ to point $P_{0}\left(x_{0}, y_{0}\right)$. We have to search for an optimal path minimizing the time associated with the trajectory $\overline{P P_{0}}$. The bang-bang control process needs a control strategy; one has to search for specific values of the slope of the trajectory $\mathrm{d} z / \mathrm{d} x$ and look for the most efficient procedure. In this fashion the half-trajectory $\overline{P Q}$ is recovered with constant time $h$, whereas path $\overline{Q P_{0}}$ has associated with it a variable time $t$ and therefore an arbitrary speed. We shall formulate the problem in one dimension for simplicity. The optical medium has refractive-index distribution $n=n(x)$. The speed of light associated with any point $P(x, z)$ inside the medium is

$$
v[x(z)]=\frac{c}{n[x(z)]},
$$

where $c$ is the speed of light in vacuum.

We define the minimum time required to optimize the trajectory $\overline{P Q P_{0}}$. The first policy is to establish an initial decision on the value of the slope trajectory, $\tan \theta$.

Following Kalaba's formulation, ${ }^{10-12}$ for the first policy to hold the half-trajectory, $\overline{Q P_{0}}$ may have an associated minimum time $t$ (optimized):

$$
\begin{aligned}
t(x, z)=\min _{\theta} & \{h+t[x+e(x, z) \cos \theta, z+e(x, z) \\
& \times \sin \theta]\}+O\left(h^{2}\right) .
\end{aligned}
$$

In Eq. (6) the trajectory $e(x, z)=v(x, z) h$ represents the variable path associated with $\overline{Q P_{0}}$ and may be a minimum. $O\left(h^{2}\right)$ is the contribution of powers of $h \gg 1$. The term between curly brackets is the minimum time required for the trajectory $\overline{P P_{0}}$ to be an optimal process.

Applying Taylor's formula in Eq. (6) and after simplification, we obtain

$$
\min _{\theta}\left\{h+e(x, z)\left[t_{x} \cos \theta+t_{z} \sin \theta\right]\right\}+O\left(h^{2}\right)=0,
$$

where $t_{x}=\partial t / \partial x, t_{z}=\partial t / \partial z$. As a first approximation, the second derivatives $\partial^{2} t / \partial x^{2}, \partial^{2} t / \partial z^{2}$ in Eq. (7) are neglected if the time of the trajectory has a linear dependence on the ray trajectory.

Dividing in Eq. (7) by $h$ and taking the limit as $h \rightarrow 0$, we get

$$
\min _{\theta}\left\{t_{x} \cos \theta+t_{z} \sin \theta\right\}=-\frac{1}{v(x, z)} .
$$

Equation (8) represents an eikonal equation. It is related to the general formulation of Maxwell's theorem for absolute instruments. ${ }^{20}$ The solution $t(x, z)$ associates a general path with an integral. The kernel is a function that depends on the vector position of a point $(x, z)$ on the ray trajectory and its first derivatives, $\mathrm{d} x / \mathrm{d} s$ and $\mathrm{d} z / \mathrm{d} s$, with respect to $s$. Here $s$ is the distance along the ray path. This statement implies that Eq. (8) has a unique solution that is a true minimum as shown by Caratheodory's theorem. ${ }^{21}$
Differentiating in Eq. (8) with respect to $\theta$ gives

$$
\tan \theta=\frac{(\partial t / \partial x)}{(\partial t / \partial z)}
$$

Equation (9) represents the slope minimizing the pathway $\overline{P P_{0}}$. For time $t$ a constant in the $z$ direction, the pathway is orthogonal to the ray trajectory coinciding with the wave front generating at $P_{0}$; and for time $t$ a constant in the $x$ direction, the pathway follows the ray trajectory.

The condition for the slope to be a minimum is that $\theta$ also be a minimum. Rearranging Eq. (9) and according to Eq. (8), we obtain

$$
\left(\frac{\partial t}{\partial x}\right)^{2}+\left(\frac{\partial t}{\partial z}\right)^{2}=\frac{n^{2}(x, z)}{c^{2}}=\frac{1}{v^{2}(x, z)},
$$

with $v(x, z)$ the speed of light propagating in a medium with refractive index $n(x, z)$ (Ref. 22).

Equation (10) is the key equation that relates the transit time $t$ to the index distribution $n(x, z)$. It is a Hamilton-Jacobi equation in two (or more) variables. In general, it has no explicit known rigorous solution. The solution depends on the optical properties of the medium under study. Solutions of the form $t=t(x, z)$ are known as geometrical time surfaces or geometrical time

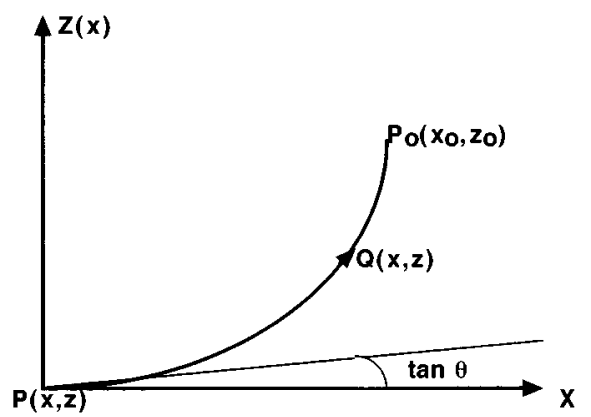

(a)

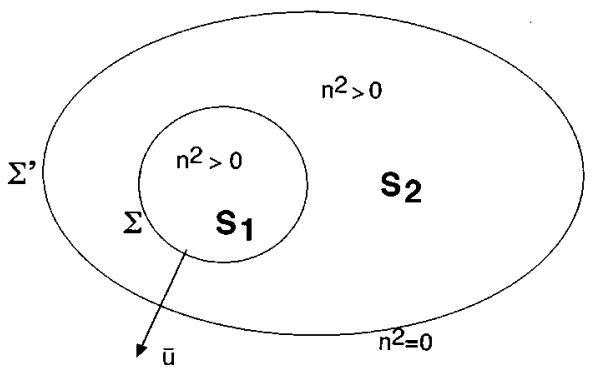

$n^{2}<0$

(b)

Fig. 2. (a) Introduction to the problem: choosing an arbitrary trajectory $z=z(x)$ ( $z$ axis, longitudinal direction; $x$ axis, transverse direction) as an arbitrary path for light propagating from an initial point $P(x, z)$ (initial time $t=0$ ) to a final point $P_{0}\left(x_{0}, z_{0}\right)$. The slope $\tan \theta$ is determined. (b) Contour $\Sigma^{\prime}$ defines an external region with surface $\mathrm{S}_{2}$, where there is propagation of light and $n^{2}(x)>0$. Contour $\Sigma$ defines an internal region with surface $\mathrm{S}_{1}$, where also $n^{2}(x)>0 . \quad \bar{u}$ is a unit vector normal to $\Sigma$. For the line contour $\Sigma^{\prime}, n^{2}(x)=0$; outside this region, $n^{2}(x)<0$ and Eq. (10) loses the meaning of a real transittime equation. 
trajectories. Their counterparts are the geometrical wave fronts from the eikonal equation. ${ }^{23}$

The following additional condition also holds:

$$
\begin{aligned}
{\left[\left(\frac{\partial e}{\partial x}\right)^{2}+\right.} & \left.\left(\frac{\partial e}{\partial z}\right)^{2}\right]+\left[\frac{e(x, z)}{v(x, z)}\right]^{2}\left[\left(\frac{\partial v}{\partial x}\right)^{2}+\left(\frac{\partial v}{\partial z}\right)^{2}\right] \\
& -2\left[\frac{e(x, z)}{v(x, z)}\right]\left[\left(\frac{\partial e}{\partial x}\right)\left(\frac{\partial v}{\partial x}\right)+\left(\frac{\partial e}{\partial z}\right)\left(\frac{\partial v}{\partial z}\right)\right]=1 .
\end{aligned}
$$

It is easy to check that Eq. (11) is analogous to Eq. (10), expressing the eikonal equation in terms of the spatial variables $e$ and $v$. After some convenient operations (see Appendix A) it also reads

$$
\left[\frac{\partial}{\partial x}\left(\ln \frac{e}{e_{0}} \frac{v_{0}}{v}\right)\right]^{2}+\left[\frac{\partial}{\partial z}\left(\ln \frac{e}{e_{0}} \frac{v_{0}}{v}\right)\right]^{2}=\frac{1}{e^{2}}
$$

where $e_{0}$ is a constant initial path having a constant initial velocity $v_{0}$ for certain given initial coordinates, $x$ $=x_{i}, z=z_{i}$. Equation (12) is a nonlinear partial differential equation whose solutions are not trivial, because to be trivial it would require additional conditions for $(e / v)$. For example, assuming $(e / v)$ independent of one of the two variables, say, $(\partial / \partial z)(\ln e / v) \approx 0$ or $(e / v)$ depending only on $\sqrt{x^{2}+z^{2}}$, which is the case of a medium with spherical symmetry, Eq. (12) simplifies to the onedimensional case that has analytical solutions. Notice that there is an equivalent equation in quantum mechanics with approximate solutions obtained by applying perturbative methods. This is the case of a classical Hamiltonian in two dimensions, the so-called Toda's potential or Toda lattice, ${ }^{24-25}$ for which the equivalent of Eq. (12) has an explicit solution. The latter is expressed as a sum of exponential functions with exponents having linear dependence on $x$ and $z$ variables. At present there is no evidence, nor available data in the literature, of optical media having such a similar refractive-index distribution. Nevertheless, establishing the correspondence between quantic potential $V$ and electromagnetic dielectric permeability $\epsilon$, interesting similarities appear between Toda's potential and Luneburg's lens, ${ }^{26}$ in which the source is placed at the surface of the unit sphere and the focus at infinity. This lens is well known in microwave optics applications as a generator of parallel rays from a virtual point source. ${ }^{27}$ It should be noted that Luneburg's lens is a generalization of Maxwell's "fish-eye" absolute instrument. Summarizing the above calculations, it seems more suitable, at least in the present study, to formulate the solution of the trajectory for the time variable $t(x, z)$ as given in Eq. (10), since application of Liouville's theorem ensures integrability of the equation. ${ }^{28}$

Let us assume that the optical medium has a defined geometry and is finite (at least in one of the two dimensions, $-x_{0} \leqslant x \leqslant+x_{0}$ ) and that the refractive index has bounded values, $n_{\mathrm{cl}} \leqslant n(x) \leqslant n_{0}$. For Eq. (10) to have a complete solution, we have to introduce the boundary conditions. Equation (10) has a physical meaning only if $n^{2}(x)>0$. Nevertheless, if the medium has a defined geometry and is finite, as in the case of an optical waveguide, there can be a contour $\Sigma^{\prime}$ limiting the region where light propagates for which $n^{2}(x)=0$. In the classical example that we are giving, this is the condition for the presence of an evanescent wave. Outside this region, $n^{2}(x)<0$, and Eq. (10) loses the physical meaning of real transit-time solutions for geometrical ray trajectories. Figure 2(b) describes two physically interesting types of boundary $\Sigma$ and $\Sigma^{\prime}$. By assumption, $\Sigma$ corresponds to the possibility that $n^{2}$ has a finite discontinuity across it, so that we have two regions, $S_{1}$ and $S_{2}$, on both sides of $\Sigma$ : in both $\mathrm{S}_{1}$ and $\mathrm{S}_{2}, n^{2}>0$. For points on line $\Sigma^{\prime}$, $n^{2}(x)=0$; outside $\Sigma^{\prime}, n^{2}(x)<0$.

Let us discuss the boundary conditions for contour $\Sigma$. At the surface of discontinuity one has to consider incident and reflected waves, to be matched by the refracted wave (the law of reflection and Snell's law have to hold). In terms of the ray transit time, we have on $\Sigma$

$$
t_{\text {in }}=t_{\text {ref }}=t_{\text {trans }},
$$

where $t_{\text {in }}$ is the ray transit time associated with the incident ray and $t_{\text {ref }}$ and $t_{\text {trans }}$ are the transit times for the reflected and the transmitted rays, respectively. One should interpret that the physical meaning of the condition in Eq. (13a) is the conservation of the eikonal phase at the surface of discontinuity.

The boundary condition for contour $\Sigma^{\prime}$ can be deduced by applying Eq. (10) to this discontinuity surface so that

$$
\left(\frac{\partial t}{\partial x}\right)^{2}+\left(\frac{\partial t}{\partial z}\right)^{2}=0
$$

One possible solution to Eq. (13b) is that $(\partial t / \partial x)^{2}$ $=(\partial t / \partial z)^{2}=0$, with $t=0$, so that at the external surface of discontinuity the transit time becomes zero. Notice that there is another solution, $(\partial t / \partial x)^{2}+(\partial t / \partial z)^{2}$ $=0$, for which the ray transit time becomes imaginary. At this point one has to recall that there is a ray-optical formalism for which the eikonal solution can be complex. This is called Felsen's general complex solution and includes the evanescent wave in the general solution. ${ }^{29}$ This leads to a general eikonal equation,

$$
\left[\left(\frac{\partial t^{(r)}}{\partial x}\right)^{2}+\left(\frac{\partial t^{(r)}}{\partial z}\right)^{2}\right]-\left[\left(\frac{\partial t^{(i)}}{\partial x}\right)^{2}+\left(\frac{\partial t^{(i)}}{\partial z}\right)^{2}\right]=\frac{n^{2}(x, z)}{c^{2}}
$$

where $t^{(r)}$ and $t^{(i)}$ are the real and the imaginary parts of the ray transit time, respectively. Equation (13c) can be interpreted in the sense of the so-called homogeneous waves, where equiphase surfaces and equiphase contours can be defined. ${ }^{30}$

Also, if $x=x_{0}, \mathrm{~d} x / \mathrm{d} z=0$, as $x=x(z)$ would be a constant. This represents a constraint or function of the position. Then

$$
\left.\frac{\partial t}{\partial x}\right|_{x=x_{0}}=\frac{n\left(x_{0}\right)}{c}
$$

Note that a more general constraint could be $r=r(z)$, with $r=\sqrt{x^{2}+y^{2}}$, which should require the formulation of the boundary conditions in cylindrical coordinates. In the next section we shall prove that $n\left(x_{0}\right)$ coincides with the ray-invariant parameter, with $x_{0}$ the turning point defined in ray trajectories in a gradient-index optical waveguide. 


\section{HOMOGENEOUS MEDIA}

Consider the simplest case of a homogeneous planar optical waveguide with refractive index $n_{0}$ defined in the $x$ region, transverse to the longitudinal axis of the waveguide $z$. Then Eq. (10) simplifies to

$$
\left(\frac{\partial t}{\partial x}\right)^{2}+\left(\frac{\partial t}{\partial z}\right)^{2}=\frac{1}{v^{2}}
$$

with $v=c / n_{0}$ a constant value, $n_{0}$ the constant refractive index of the medium, and $c$ the speed of light.

The solution of Eq. (14) is the simplest one for a partial differential equation of hyperbolic type. ${ }^{31}$ Since in the $x$ direction the time is a constant, $\partial t / \partial x=0$, then in Eq. (14) the characteristics are straight lines:

$$
\begin{aligned}
& t-(z / v)=\xi, \\
& t+(z / v)=\eta,
\end{aligned}
$$

with $\xi$ and $\eta$ constants. Equations (15a) and (15b) amount to global constraints for the system (defining a family of lines or linear trajectories).

We emphasize this idea with the notation

$$
t \rightarrow t \pm(z / v) \text {. }
$$

Also, we note that Eqs. (15a) and (15b) represent Fermat's principle:

$$
t_{\min }= \pm \int_{z_{\min }}(1 / v) \mathrm{d} z
$$

with the initial condition $t(z, 0)=0$.

Let us recall here the transit time for the ray trajectory in a homogeneous planar, or slab, waveguide, as predicted by the geometrical optics approximation ${ }^{32}$.

$$
t=\left(\frac{n_{0}}{c}\right)\left(\frac{1}{\cos \theta_{z}}\right) \int_{z_{\min }} \mathrm{d} z,
$$

with $0 \leqslant \theta_{z}(z) \leqslant \theta_{C}$, and $\theta_{C}$ the complementary of the critical angle. An initial condition over the slope is $\theta(z)$ $=\theta\left(z_{0}\right), z_{0}$ constant. The initial strategy in this case reduces to selection of the critical angle. Two main parameters are introduced in the process of searching for a minimum pathway: (1) angle $\theta$ between the trajectory and the axial direction and (2) longitudinal variable $z$. The slope of the trajectory as defined in Eq. (9) is also a constant. The initial global constraint is $x=z_{0} \tan \theta$.

\section{GRADIENT-INDEX OPTICAL WAVEGUIDES}

Consider an arbitrary gradient-profile planar waveguide with refractive-index distribution following a $p$ law:

$$
n^{2}(x)=n_{0}^{2}\left[1-2 \Delta(x / \rho)^{p}\right],
$$

with $p \geqslant 1, \Delta$ the height of the profile, $\rho$ the half-width of the waveguide, and $n_{0}$ the refractive index of the core.

In this case Eq. (10) reads

$$
\left(\frac{\partial t}{\partial x}\right)^{2}+\left(\frac{\partial t}{\partial z}\right)^{2}=\frac{n^{2}(x)}{c^{2}}=\frac{1}{v^{2}(x)},
$$

with $n(x)$ following the law defined in Eq. (19).
Equation (20) is the equation of the characteristics for the optimized time of the trajectory. It depends on the variable $x$ (cross section) and the $z$ longitudinal direction of the waveguide. The type of solution depends on the value of $p$.

Operating on Eq. (20) and taking into account the constraints on the system, we arrive at

$$
\frac{\mathrm{d} t}{\mathrm{~d} x}= \pm\left(\frac{1}{c}\right) \frac{n(x)}{\left[1+z^{\prime 2}\right]^{1 / 2}} z^{\prime},
$$

with $z^{\prime}=\mathrm{d} z / \mathrm{d} x$ the slope of the trajectory, $z=z(x)$. This global constraint represents a one-dimensional policy since we are defining $z$ with a single variable. One has to evaluate $z=z(x)$ for each particular trajectory, say, for each particular medium. Intuitively, function $z$ $=z(x)$ defines the ray trajectory as defined by the geometrical optics approximation. The correct formulation provides identical results.

Let us analyze Eq. (21). The Euler-Lagrange equation becomes, in this case,

$$
\frac{\mathrm{d}^{2} t}{\mathrm{~d} x^{2}}=\left(\frac{1}{c}\right) \frac{\mathrm{d}}{\mathrm{d} x}\left[\frac{n(x)}{\left(1+z^{\prime 2}\right)^{1 / 2}} z^{\prime}\right]=0,
$$

which implies that

$$
\frac{n(x)}{\left(1+z^{\prime 2}\right)^{1 / 2}} z^{\prime}=c_{1},
$$

with $c_{1}$ a constant. Then Eq. (23) is

$$
z(x)=c_{1} \int \frac{1}{\left[n^{2}(x)-c_{1}^{2}\right]^{1 / 2}} \mathrm{~d} x .
$$

Equation (24) coincides with the ray-path parameter characterizing ray propagation in graded-profile waveguides with the condition

$$
c_{1}=\bar{\beta}
$$

In Eq. (25), $\bar{\beta}$ is the ray-invariant or ray-constant parameter. ${ }^{33}$ Equation (24) defines the slope of the trajectory $z^{\prime}$. Defining the first strategy as $z=z(x)$ (and $z^{\prime}$ ), we can reasonably start from Eq. (22) to obtain analytical and numerical solutions for the minimization of the quadratic functional. The Euler equation [Eq. (22)] together with the boundary condition [Eq. (25)] assigns a unique solution that gives the absolute minimum of the quadratic functional. The procedure is a variational technique, e.g., the Rayleigh-Ritz method. ${ }^{34}$

The time minimizing the longitudinal trajectory for the light inside the waveguide is

$$
t(z)=t^{\prime}\left(z_{p}\right)+\frac{1}{c} \int \frac{n(z)}{\left(1+z^{\prime 2}\right)^{1 / 2}} \mathrm{~d} z .
$$

Notice that in Eq. (26) we are integrating over the variable trajectory $x=x(z)$ and that the constant of integration $t^{\prime}\left(z_{p}\right)$ is an initial condition (e.g., the value for $\mathrm{d} t / \mathrm{d} z$ is zero) and holds for $z=z_{p}$. For example, for symmetrical profiles, $z_{p}$ represents the half-period (longitudinal section) of the trajectory between two turning points. This is not a restrictive condition, since for asymmetrical profiles one could apply the appropriate initial condition for an entire period. The first property to be 
checked for the integrability of Eq. (26) is that the convergence of the solution be ensured. This will depend on the form of $n(z)$ and the behavior of $z^{\prime}$. Similarly, for the $x$ direction,

$$
t(x)=\frac{1}{c} \int^{x} L(x, u) \mathrm{d} x,
$$

with

$$
L(x, u)=\frac{n(x)}{\left[1+u^{2}(x)\right]^{1 / 2}},
$$

where $u(x)=1 / z^{\prime} . \quad u(x)$ has to be a continuous function with continuous first derivative $u^{\prime}(x)$. We notice that $L(u, x)$ is a nonlinear differential operator. It has the physical meaning of a differential pathway. Also, the condition $u\left(x_{t p}\right)=0$ holds (it is not necessarily a boundary condition). The point $x_{t p}$ is similar to the turning point in the $x=x(z)$ trajectory. Explicit integration of Eq. (27) depends on a law-clad profile. For example, for $p>2$, Eq. (27) could diverge, and one would need to adjust the physical parameters of the waveguide.

Equation (27) is one of the forms of the so-called problem of Mayer ${ }^{35}$ commonly found in terminal control problems. In the present study, $t(x)$ is, moreover, a solution with fixed end points (defining the dimensions of the waveguide); it has approximate analytical or numerical solutions.

\section{PARABOLIC PROFILE AND NUMERICAL RESULTS}

Assume that $p=2$ in Eq. (19). Then Eq. (24) has an immediate analytical solution:

$$
z(x)=\left(\frac{\bar{\beta} \rho}{n_{0} \sqrt{2 \Delta}}\right) \arcsin \left(\frac{x}{x_{t p}}\right),
$$

with slope

$$
z^{\prime}(x)=\frac{\sqrt{2} \bar{\beta} \rho}{2 n_{0} x_{t p} \sqrt{\Delta\left[1-\left(x / x_{t p}\right)^{2}\right]}} .
$$

Similarly, Eq. (29) determines $x=x(z)$ and $u=u(x)$.

Figure 3 shows the refractive-index-profile distribution in the $X-Z$ plane representing $n[x, x(z)]=n(x) n[x(z)]$ for the following physical parameters: $\rho=3 \mathrm{~mm}, \Delta$ $=0.00783, n_{0}=1.527$. To assign a numerical value to $\bar{\beta}$, one should take into account Eq. (25). Since $\bar{\beta}$ represents the ray-invariant parameter, as defined in the geometrical optics approximation, it has to obey $1.50 \leqslant \bar{\beta}$ $\leqslant 1.527$, where 1.50 is the refractive index of the cladding and 1.527 the refractive index of the core. We have considered $\bar{\beta}=n\left(x_{t p}\right)=1.515$ in the present study. ${ }^{36}$ For these values, $x_{t p}=3 \mathrm{~mm}$ and $z_{p}=74.73 \mathrm{~mm}$. Figure 4 displays a three-dimensional plot of the trajectories $z=z(x)$ and $x=x(z)$ as given in Eqs. (24) and (25). By representing both functions simultaneously, one appreciates a complete description of the spatial ray geometry. Similarly, Fig. 5 gives the shape of the slope of the trajectory, $z^{\prime}=z^{\prime}(x, z)$ [see Eq. (30)], showing the constant behavior in the $z$ direction as expected.
Considering $z=z(x)$ as the initial policy and $z^{\prime}$ as the global constraint, one can determine numerically the optimized time $t(z)$ and $t(x)$.

The results for the time $t(z)$ with the above physical parameters are shown in Fig. 6(a). This function fits a

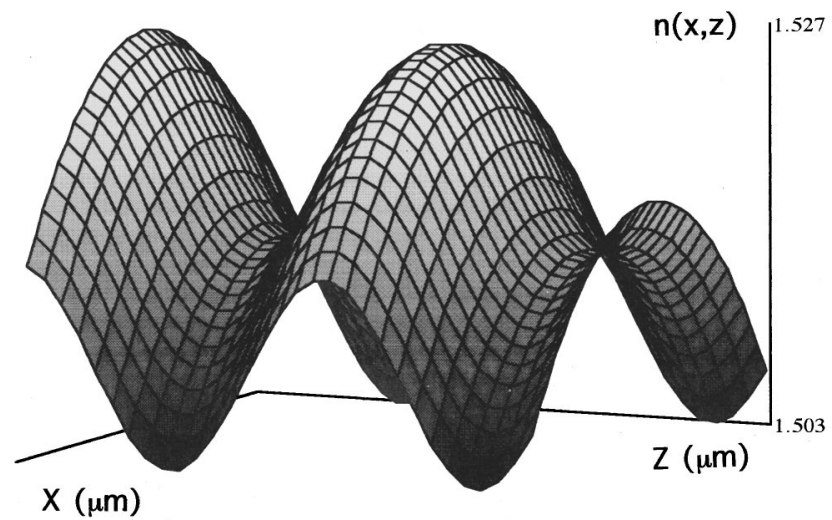

Fig. 3. Refractive-index distribution $n[x, x(z)]=n(x) n[x(z)]$ in the $X-Z$ plane for a planar optical waveguide with $p=2$ [see Eq. (19)]. Physical parameters are $n_{0}=1.527, \Delta=0.0078, \rho$ $=3 \mathrm{~mm}$. The refractive-index distribution is shown along both the transverse and the longitudinal trajectories according to Eq. (29). The sinusoidal dependence in the $z$ direction can be seen.

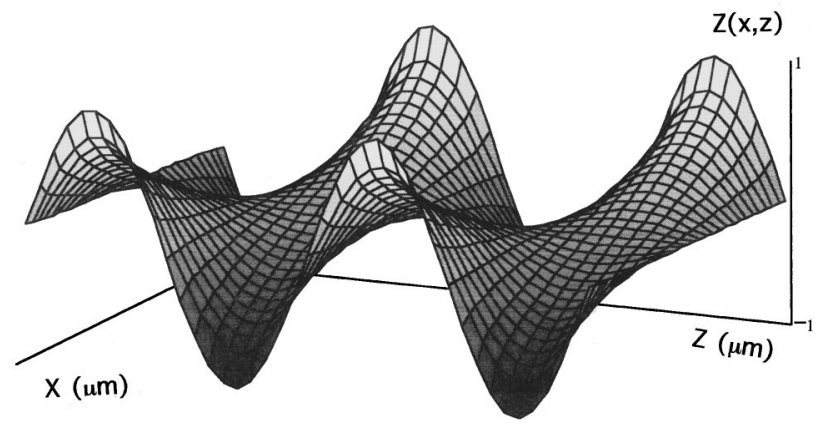

Fig. 4. A three-dimensional plot of the two trajectories $z(x)$ and $x(z)$ as given from Eqs. (24) and (25), giving a complete description of the spatial ray geometry. The vertical axis is in arbitrary units. $z$ ranges from 2 to $300 \mu \mathrm{m}$, and $-2 \leqslant x \leqslant+2(\mu \mathrm{m})$. $\bar{\beta}=1.151$ is the ray-invariant parameter. See text for details.

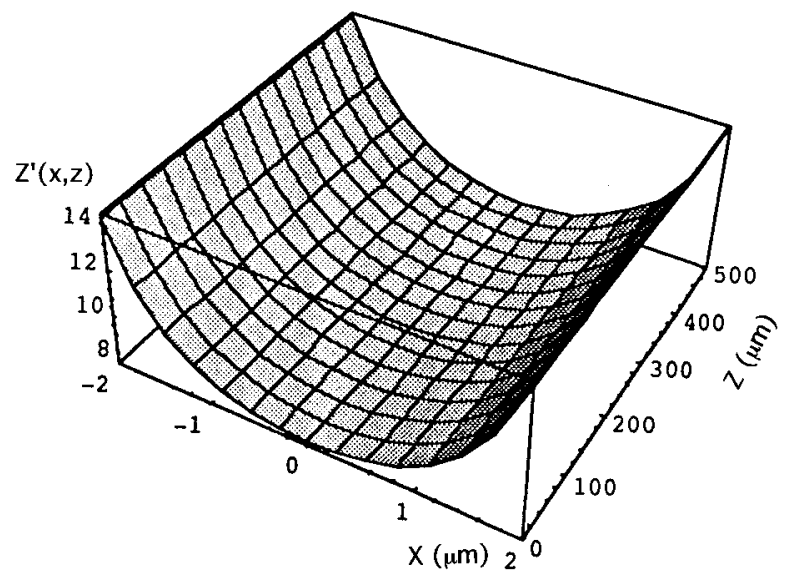

Fig. 5. Slope of the trajectory displayed in Fig. $4, z^{\prime}=\mathrm{d} z / \mathrm{d} x$ showing, a gradient in the $x$ direction and constant values in the $z$ direction. 


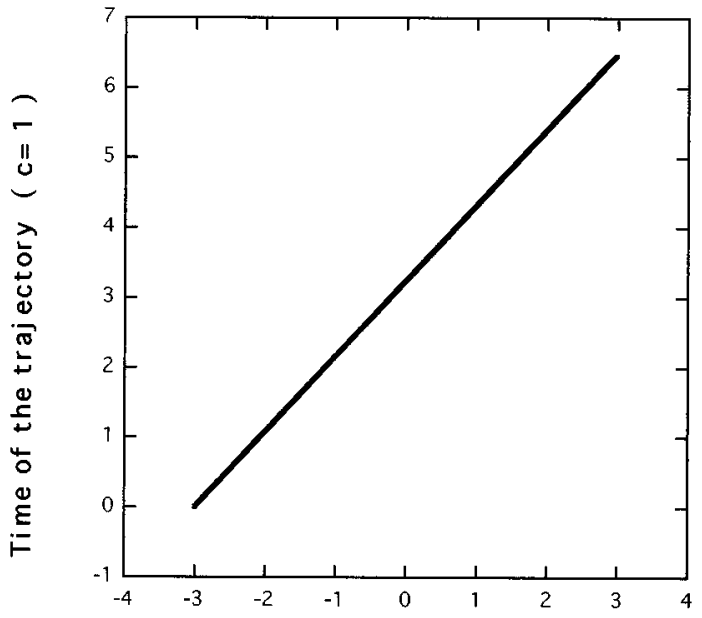

Ray trajectory $x=x(z)($ micrometers $)$

(a)

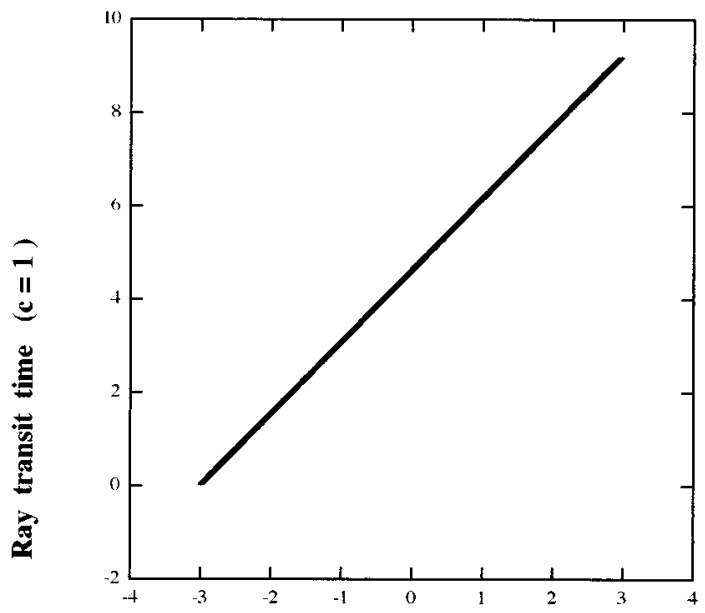

Ray trajectory $x=x(z)$ (micrometers)

(b)

Fig. 6. (a) Linear dependence of the time of the trajectory $t$ $=t(z)$ as given by the application of dynamic programming [see Eq. (26)] with $x=x(z)$. For simplicity we consider $c=1$. (b) Ray transit time as predicted by the geometrical optics approximation. The inverse of the local speed $n(x) / c$ ( $c$, free-space speed of light) is integrated along the curved ray path $x=x(z)$ $(c=1)$.

linear variation for points of the sinusoidal trajectory $x$ $=x(z)$. This result is comparable to the ray transit time as predicted by the geometrical optics approximation, ${ }^{32}$ whose distribution is shown in Fig. 6(b). The corresponding slope $[\partial t(x, z)] / \partial z$ associated with $t(z)$ is given in Fig. 7 (a) for the actual trajectory $x=x(z)$. The figure shows the gradual change of the slope for different points of the trajectory, exhibiting a skewlike variation. For the sake of comparison, Fig. 7(b) displays the corresponding slope associated with the ray transit time. The shape of these two functions depends dramatically on the physical parameters of the waveguide, with special influence on $z_{p}$ (the value for which the trajectory has zero slope). The linear time distribution $t(z)$ obtained through dynamic programming appears to have a smaller slope than the one corresponding to the transit time.
Similarly, Fig. 8 gives the variation of the $t(x)$ function. This exhibits linear dependence, as it does for $t=t(z)$. Here the slope $[\partial t(x, z)] / \partial x$ coincides with the operator $L(x, u)$ as defined in Eq. (28). These results are presented in Fig. 9. The figure shows how the time compensates across the transverse section of the waveguide. It is equivalent to the optimum profile given by Snyder and Love $^{37}$ for the transit time or equalization. We notice that the minimum value $L_{\min }=L(0, u=$ const.) equals the ray-invariant parameter $\bar{\beta}$, as expected for an optimized profile. It is remarkable that the interval $L_{\max }-L_{\min }=0.01181$ (for $\bar{\beta}=1.515$ ) is small but sufficient for estimating the pulse width: $t_{\max }-t_{\min }=\Delta t$. The variation of the time compensation concerning $\bar{\beta}$ for the boundary points of the waveguide is negligible but becomes much more appreciable for points on the axis, where the highest value is obtained for $\bar{\beta}=1.50$ (coinciding with the refractive-index distribution of the cladding).

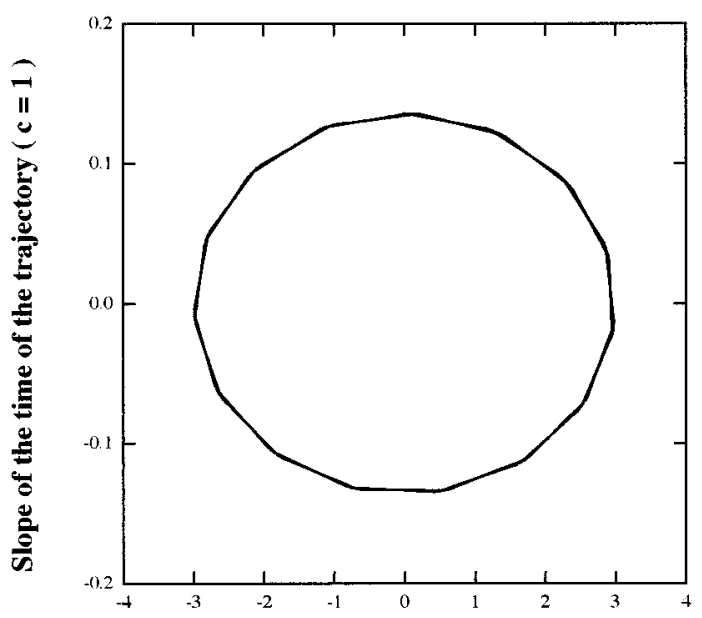

Ray trajectory $x=x(z)$ (micrometers)

(a)

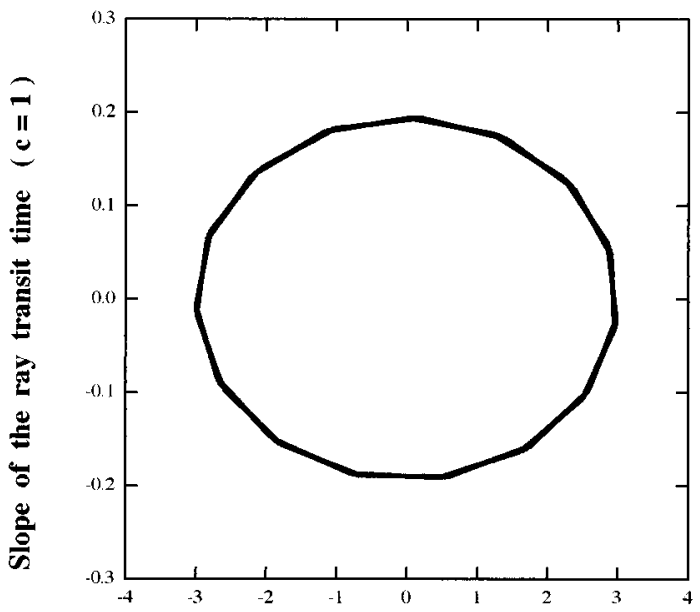

Ray trajectory $x=x(z)$ (micrometers)

(b)

Fig. 7. (a) Slope associated with time $t=t(z)$ as given by dynamic programming [see Fig. 6(a)]. (b) Slope of the ray transit time as displayed in Fig. 6(b). 


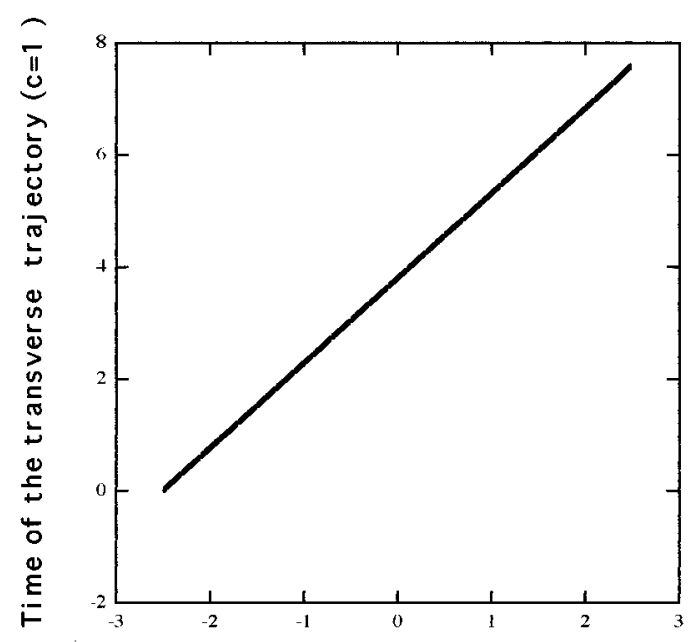

Cross section of the waveguide (micrometers)

Fig. 8. Time distribution across the transverse trajectory (cross section of the waveguide) according to dynamic programming [see Eqs. (27) and (28)].

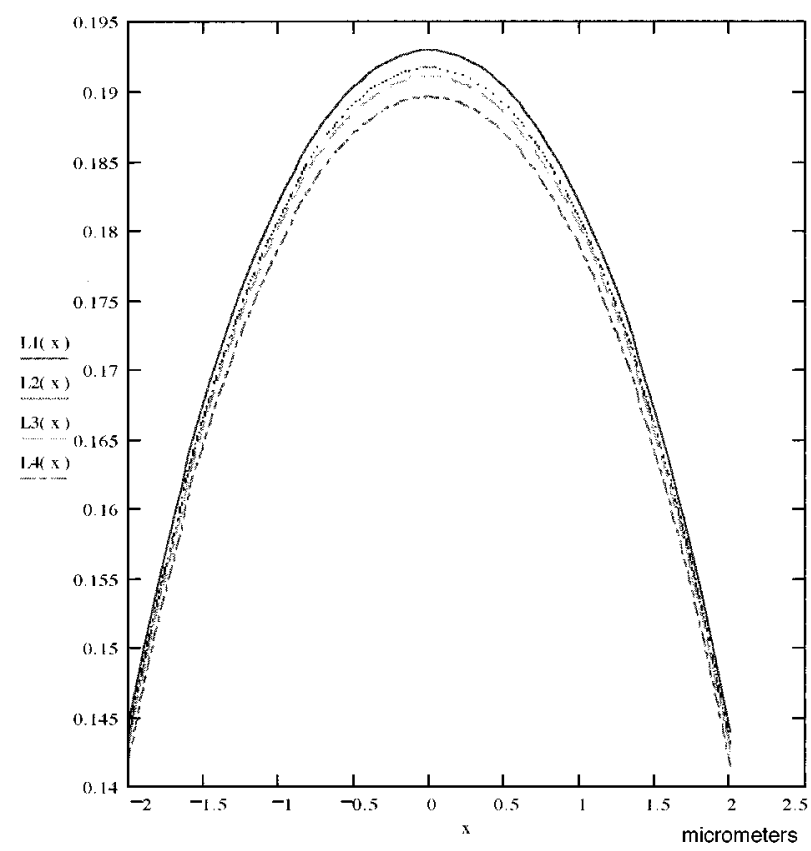

Fig. 9. Slope of the time variation represented in Fig. 8, coinciding with Eq. (28), the differential operator $L(x, u)$. This figure indicates how the time compensates across the transverse section of the waveguide. It is equivalent to the optimum profile given by Snyder and Love ${ }^{37}$ for the transit time or equalization. Minimum equalization corresponds to the waveguide axis (minimum local speed). Maximum equalization corresponds to the boundary of the guide (maximum local speed). We represent $L(x, u)$ for four values of $\bar{\beta}$ : (1) solid curve, $\bar{\beta}=1.500$; (2) dotted curve, $\bar{\beta}=1.510$; (3) dashed curve, $\bar{\beta}=1.515$; (4) dasheddotted curve, $\bar{\beta}=1.527$. See text for details.

Thus the maximum spreading of the pulse corresponds to $\bar{\beta}=n_{\mathrm{cl}}$ and the minimum to $\bar{\beta}=n_{0}$. Nonetheless, the differences are not dramatic, because we are considering an optimum profile $(p=2)$. As the treatment holds for arbitrary $n(x)$ distribution, it could be generalized to any optical media, provided that the convergence of the solu- tion is ensured. The extension to arbitrary profile $n$ $=n(x, y)$ (two-dimensional problem) is also possible with the restrictions mentioned above. As a proof of consistency, one may check that with use of the numerical result for $\partial t / \partial x$ and $\partial t / \partial z$ together with the $n(x)$ profile, Eq. (20) holds. Evaluating thus one finds values of the order of 2.29-2.33 for both sides of Eq. (20). The discrepancies are due to computational round-off errors.

\section{DISCUSSION AND CONCLUSIONS}

Dynamic programming is a powerful technique for solving optimization problems in many fields of science. The optimal procedure consists of introducing an initial condition that determines the final state of the system. In the optical problem of defining minimum or optimized pathways (time minimization), dynamic programming provides immediate solutions in the form of equal-time surfaces, $t=t(x, z) \cong$ const. Its physical meaning is that of wave fronts generated at the initial point and zero time (zero instant). These basic ideas were first noted by Kalaba, ${ }^{10}$ who demonstrated that the mathematical procedure optimizing pathways under an initial slope of the trajectory is equivalent to solving an eikonal-type equation.

The main result of this paper is the development of the transit-time equation based on a dynamic-programming approach in the problem of light propagation in arbitrary optical media.

We have proven the simplicity of this technique by studying various cases of waveguides that have gradientindex profiles. The partial differential equation in terms of $\partial t / \partial x$ and $\partial t / \partial z$ for arbitrary refractive-index distribution is a Hamilton-Jacobi type and has Euler-Lagrange implicit solutions. It automatically gives the initial policy- $z=z(x)$, trajectory, and $z^{\prime}=\mathrm{d} z / \mathrm{d} x$, slope-as global constraints that determine the final state of the system. The integral form of $z(x)$ equals the one from the eikonal equation, with the additional condition that the constant of integration be the ray-invariant $\bar{\beta}$. The solutions for $t=t(z)$ have behavior equivalent to the ray transit time predicted by the geometrical optics approximation. The numerical estimates carried out demonstrate this statement clearly. Moreover, the slope of the trajectory $t=t(x)$ has meaning similar to the optimum profile distribution under the geometrical optics approximation. The slope represents the transit-time variation with the ray invariant $\bar{\beta}$. The results indicate that the value of the differential operator $L(x, u)$ at the origin (axial direction) coincides with $\bar{\beta}=1.151$ (as in the present study). This curve displays the time equalization for specific trajectories and may be useful for designing specific optical media. It also provides numerical values for the pulse spread. The inverse problem might be analyzed-for example, searching for optimized optical media (fastest propagation of the luminous signal with the lowest distortion). Possible restrictions come from the analytical behavior of the integrals, and one needs to check the convergence of the solution. For example, we analyzed additional results for the law-clad profile $p$ $=4$ (not discussed here for brevity), obtaining divergence for some values of the physical parameters. The method 
is applicable only in the context in which Fermat's principle holds. It appears to have an equivalence in quantum mechanics. In this context, solutions of the Hamilton-Jacobi-type equations are assured as per Liouville's theorem. Exact solutions are valuable only for specific potentials (having approximately an equivalence with the squared refractive-index distribution). These aspects are now under investigation and will be presented in forthcoming papers.

\section{APPENDIX A}

We look for a compact form of Eq. (11):

$$
\begin{aligned}
{\left[\left(\frac{\partial e}{\partial x}\right)^{2}+\right.} & \left.\left(\frac{\partial e}{\partial z}\right)^{2}\right]+\left[\frac{e(x, z)}{v(x, z)}\right]^{2}\left[\left(\frac{\partial v}{\partial x}\right)^{2}+\left(\frac{\partial v}{\partial z}\right)^{2}\right] \\
& -2\left[\frac{e(x, z)}{v(x, z)}\right]\left[\left(\frac{\partial e}{\partial x}\right)\left(\frac{\partial v}{\partial x}\right)+\left(\frac{\partial e}{\partial z}\right)\left(\frac{\partial v}{\partial z}\right)\right]=1 .
\end{aligned}
$$

Dividing both sides of Eq. (A1) by $1 / e^{2}$, we get

$$
\begin{gathered}
{\left[\left(\frac{\partial \ln e}{\partial x}\right)^{2}+\left(\frac{\partial \ln e}{\partial z}\right)^{2}\right]+\left[\left(\frac{\partial \ln v}{\partial x}\right)^{2}+\left(\frac{\partial \ln v}{\partial z}\right)^{2}\right]} \\
-2\left[\frac{\partial \ln e}{\partial x} \frac{\partial \ln v}{\partial x}+\frac{\partial \ln e}{\partial z} \frac{\partial \ln v}{\partial z}\right]=\frac{1}{e^{2}}
\end{gathered}
$$

Applying relations of vectorial analysis Eq. (A2) produces

$$
\left|e \bar{\nabla}\left(\ln \frac{e}{v}\right)\right|^{2}=1 .
$$

One can object that in Eq. (A3) $e / v$ is not a dimensionless quantity (since it is not mathematically correct to represent a logarithm with dimensions). To avoid this inconsistency we introduce constant values of $e$ and $v-e_{i}$ and $v_{i}$-for certain given initial coordinates $x=x_{i}, z=z_{i}$, $e_{0}=e\left(x_{i}, z_{i}\right), v_{0}=v_{i}\left(x_{i}, z_{i}\right)$. The introduction of these two constants does not alter Eq. (A3). Then

$$
\left|e \bar{\nabla} \ln \left(\frac{e}{e_{0}} \frac{v_{0}}{v}\right)\right|^{2}=1,
$$

or

$$
\left[\frac{\partial}{\partial x} \ln \left(\frac{e}{e_{0}} \frac{v_{0}}{v}\right)\right]^{2}+\left[\frac{\partial}{\partial z} \ln \left(\frac{e}{e_{0}} \frac{v_{0}}{v}\right)\right]^{2}=\frac{1}{e^{2}},
$$

which is the same as Eq. (12).

\section{ACKNOWLEDGMENTS}

This work was supported in part by the School of Optometry of the University of Missouri-St. Louis (UMSL). A UMSL research grant is also gratefully acknowledged. We also thank Jerry Christensen, Dean, School of Optometry, USML, for support during the initial stages of this research program. Additional financial assistance from the Spanish Ministry of Education and Science (DGICYT) under project PR94-362 to M. L. Calvo is also acknowledged. Partial results were presented at the OSA Annual Meeting (Portland, Oregon, October 1995) and at the ICO-XVII Congress (Taejon, Korea, August 1996). The authors also thank an anonymous referee for a careful re- view and R. F. Alvarez-Estrada and G. F. Calvo for helpful suggestions and discussions.

M. L. Calvo can be contacted as follows: tel, 341-3944684; fax, 341-394-4683; e-mail, mlcalvo@eucmax.sim. ucm.es.

\section{REFERENCES AND NOTES}

1. R. K. Lagu and R. V. Ramaswamy, "A variational finite difference method for analyzing channel waveguides with arbitrary index profiles," IEEE J. Quantum Electron. QE-22, 968-978 (1986).

2. A. Sharma and P. Bindal, "Variational analysis of diffused planar and channel waveguides and directional couplers," J. Opt. Soc. Am. A 11, 2244-2248 (1994).

3. A. Sharma, D. V. Kumar, and A. K. Ghatak, "Tracing rays through graded-index media: a new method," Appl. Opt. 21, 947-987 (1982).

4. B. D. Stone and G. W. Forbes, "Optimal interpolants for Runge-Kutta ray tracing in inhomogeneous media," J. Opt. Soc. Am. A 7, 248-254 (1990).

5. J. Puchalsky, "Numerical determination of continuous ray tracing: the four-component method," Appl. Opt. 33, 1900-1906 (1994).

6. K. B. Wolf and G. Krötzen, "Geometry and dynamics in refracting systems," Eur. J. Phys. 16, 14-20 (1995).

7. A. Ghatak, E. Sharma, and J. Kompella, "Exact ray paths in bent waveguides," Appl. Opt. 27, 3180-3184 (1988).

8. A. Ghatak and E. G. Sauter, "The harmonic oscillator problem and the parabolic index optical waveguide I. Classical and ray optics analysis," Eur. J. Phys. 10, 136-143 (1989).

9. E. G. Sauter and A. K. Ghatak, "The harmonic oscillator problem and the parabolic index optical waveguide II. Quantum mechanical and wave optical analysis," Eur. J. Phys. 10, 144-150 (1989).

10. R. Kalaba, "Dynamic programming, Fermat's principle, and the eikonal equation," J. Opt. Soc. Am. 51, 1150-1151 (1961). See also V. Lakshminarayanan and S. Varadharajan, "Dynamic programming, Fermat's principle, and the eikonal equation-revisited," J. Optimization Theor. Appl. (to be published).

11. R. E. Bellman, Dynamic Programming (Princeton U. Press, Princeton, N.J., 1957).

12. V. Lakshminarayanan, S. Varadharajan, and M. L. Calvo, "A note on the applicability of dynamic programming to waveguide problems," in Photonics '96: Proceedings of the International Conference on Fiber Optics and Photonics, J. P. Raina and P. R. Vaya, eds. (Tata McGraw-Hill, New Delhi, 1977), Vol. 1, pp. 209-214.

13. R. E. Bellman and R. Kalaba, "Dynamic programming, invariant imbedding and quasi-linearization: comparison and interconnections," in Computing Methods in Optimization Problems, A. V. Balakrishnan and L. W. Neustadt, eds. (Academic, New York, 1964), pp. 135-145.

14. S. E. Dreyfus, Dynamic Programming and the Calculus of Variation (Academic, New York, 1965).

15. R. E. Bellman and R. Vasudevan, Wave Propagation. An Invariant Imbedding Approach (Reidel, Dordrecht, The Netherlands, 1986).

16. S. E. Dreyfus and A. M. Law, The Art and Theory of Dynamic Programming (Academic, New York, 1977).

17. R. E. Bellman, R. Kalaba, and G. M. Wing, "Invariant imbedding and mathematical physics I. Particle process," J. Math. Phys. 1, 280-308 (1960).

18. R. E. Bellman, Introduction to the Mathematical Theory of Control Processes. Linear Equations and Quadratic Criteria (Academic, New York, 1967), Chaps. 3 and 4.

19. R. E. Bellman and S. E. Dreyfus, Applied Dynamic Programming (Princeton U. Press, Princeton, N.J. 1962), Chap. VIII.

20. M. Born and E. Wolf, Principles of Optics, 6th ed. (Pergamon, Oxford, 1984), Sec. 4.2

21. Caratheodory's theorem establishes the connection between Fermat's principle and the calculus of variation de- 
fining precise extremals. See C. Caratheodory, Geometrische Optik (Springer, Berlin, 1937). See also Ref. 20, App. 1 , pp. $719-737$.

22. M. L. Calvo and V. Lakshminarayanan, "Dynamic programming: an alternative approach to light propagation in arbitrary optical media," in 17th Congress of the International Commission for Optics: Optics for Science and New Technology, J. S. Chang, J. H. Lee, S. Y. Lee, and C. H. Nam, eds., Proc. SPIE, 2778, 294-295 (1996).

23. See, for example, Ref. 20, p. 111.

24. R. F. Alvarez-Estrada, Department of Theoretical Physics, Faculty of Physical Sciences, Universidad Complutense, 28040 Madrid, Spain, June 1996 (personal communication).

25. Toda's potential, also called the Toda lattice, is a wellknown solution of Eq. (12) in the field of quantum mechanics and nonlinear waves. See M. Toda, "Vibration of a chain with nonlinear interaction," J. Phys. Soc. Jpn. 22, 431-436 (1967); "Wave propagation in anharmonic lattices," J. Phys. Soc. Jpn. 23, 501-506 (1967); A. Khare and R. K. Bhaduri, "Exactly solvable noncentral potentials in two and three dimensions," Am. J. Phys. 62, 1008-1014 (1994).

26. R. K. Luneburg, The Mathematical Theory of Optics (U. California Press, Berkeley, Calif., 1964), p. 180.

27. S. Cornbleet, Microwave Optics, The Optics of Microwave Antenna Design (Academic, New York, 1976), pp. 127-132. See also Sec. 2.6.

28. E. T. Whittaker, A Treatise on the Analytical Dynamics of Particles and Rigid Bodies, 4th ed. (Cambridge U. Press, London, 1964), Chap. X, p. 281.

29. L. B. Felsen and M. Marcuwitz, Radiation and Scattering of Waves (Prentice-Hall, Englewood Cliffs, N.J., 1973).
30. Some interesting formalism related to the so-called homogeneous-wave solution can be found in S. Solimeno, B. Crosignani, and P. Di Porto, Guiding, Diffraction and Confinement of Optical Radiation (Academic, New York, 1986), pp. 63-66.

31. J. Mathews and R. L. Walker, Mathematical Methods of Physics (W. A. Benjamin, New York, 1970), Sec. 8-2.

32. A. W. Snyder and J. D. Love, Optical Waveguide Theory (Chapman \& Hall, London, 1983), Sec. 1-9.

33. A. W. Snyder and J. D. Love, Optical Waveguide Theory (Chapman \& Hall, London, 1983), Sec. 1-8.

34. Although the Rayleigh-Ritz method is discussed in the literature in relation to variational principles, here we introduce applications in the context of dynamic programming. See, for example, Ref. 15, Chap. X, Sec. 3.

35. G. A. Bliss, Lectures on the Calculus of Variation (U. of Chicago Press, Chicago, Ill., 1959).

36. The constant of integration $\bar{\beta}$ plays an interesting role in the dynamic-programming formulation. It ensures the similarity between the trajectory $z(x)$ given in Eq. (24) and the ray propagation in graded-index-profile waveguides under the geometrical optics approximation. Even if this similarity were to be ignored, there should appear values of $\bar{\beta} \geqslant n_{0}, n_{0}$ being the refractive index of the core, for which the integral in Eq. (24) diverges. This indicates that only bound trajectories (bound rays) are convergent solutions of Eq. (24).

37. A. W. Snyder and J. D. Love, Optical Waveguide Theory (Chapman \& Hall, London, 1983), Sec. 3-2. 